\title{
Efficient Incremental Mining of Contrast Patterns in Changing Data
}

\author{
James Bailey and Elsa Loekito \\ Department of Computer Science and Software Engineering \\ The University of Melbourne, Australia
}

Key words: Data mining, Contrast patterns, Databases

\section{Introduction}

A contrast pattern, also known as an emerging pattern [7], is an itemset whose frequency differs significantly between two classes of data. Such patterns describe differences between datasets and have been shown to be useful for building powerful classifiers $[11,9,2,8]^{1}$. Incrementally mining them in changing data is very important, where transactions can be inserted and deleted and mining needs to be repeated after changes occur. When the changes are small, the previously mined contrast patterns should be reused where possible, to compute the new patterns. A primary example of changing data is a data stream - a sequence of continuously arriving transactions (or itemsets). Mining of contrast patterns in a data stream is useful for stream classification [2] and network traffic change detection [4]. Work in [10] presented an algorithm to incrementally mine contrast patterns, but is oriented to updates of a single type. When a dataset changes due to insertion and deletion together, the efficiency of [10]'s approach is reduced, due to redundant computations. In this paper, we present a new algorithm that addresses the scenario of incrementally mining contrast patterns in response to simultaneous insertion and deletion. Our ap-

Email addresses: (jbailey,eloekito)@cs.mu.oz.au (James Bailey and Elsa Loekito)

${ }^{1}$ See http://www.cs.wright.edu/ gdong/EPC.html for a comprehensive bibliography

Preprint submitted to Information Processing Letters

January 26, 2010 
proach can be applied to any evolving dataset, but we particularly focus on data streams, a popular type of dataset for data mining (e.g. [6, 1]). The patterns of contrast correspond to itemsets which appear in the more recent transactions and not in the less recent ones.

A sliding window model [5] is a natural choice for determining which data to include for stream contrasts. Here, efficient incremental maintenance of the patterns in the sliding window is important. Arrival of new transactions deletes some of the oldest transactions from the window. This simultaneous transaction insertion and deletion may result in some interactions between these updates. The existing incremental technique for mining contrast patterns [10], performs well when changes of a single type occur in the input data, but has drawbacks when changes of multiple types simultaneously occur.

Contributions: We propose a new efficient technique for incrementally mining contrast patterns in scenarios where simultaneous insertions and deletions occur, such as in a data stream. We experimentally show it can deliver substantial speedups over the previous approach of [10].

\section{Preliminary Definitions}

Assume a database $D$, with attributes $\left\{A_{1}, A_{2}, \ldots, A_{n}\right\}$. Attribute $A_{i}$ is defined by some values $\operatorname{domain}\left(A_{i}\right)$. The set of all items $I$ is the aggregate of all such domain values across attributes. $I=\bigcup_{i=1 . . n}$ domain $\left(A_{i}\right)$. An itemset is a set of items in $I$. A dataset is a set of transactions, where each transaction is an itemset. For itemsets, $P$ and $Q, Q$ is a superset of $P$, or $Q$ contains $P$, iff every item in $P$ is also in $Q$. Given two sets of itemsets, $X$ and $Y, X \cap_{B} Y$ denotes the border intersection of $X$ and $Y$, namely all elements of $X$ which are contained in at least one element of $Y$. The support of an itemset $S$ in $D, \operatorname{support}_{D}(S)$, is the

number of transactions which contain $S$, divided by the number of transactions 


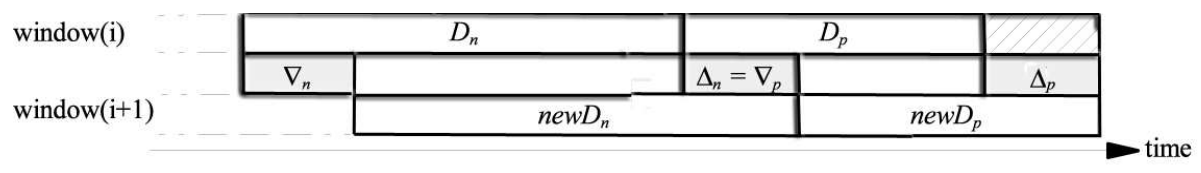

Figure 1: Sliding Window Model $\left(\left|\nabla_{n}\right|=\left|\Delta_{n}\right|=\left|\nabla_{p}\right|=\left|\Delta_{p}\right|=\delta\right)$

in $D$. A principal type of contrast pattern is the emerging pattern (EP) [7], which is an itemset whose support increases significantly from one dataset, labeled as the negative class, to another, labeled as the positive class. Let $D_{p}$ and $D_{n}$ be the positive and negative classes. The growth rate of $S$ is the ratio of its support in $D_{p}$ over that in $D_{n}$, i.e. $\frac{\text { support }_{D_{p}}(S)}{\text { support }_{D_{n}}(S)}$. We will focus on a particular type of pattern, known as the Jumping Emerging Pattern (JEP)[7], which is an EP with an infinite growth rate (i.e. support $_{D_{n}}(S)=0$ ) and can capture sharp contrasts. A JEP is minimal if it is not a superset of other JEPs. Focusing on mining only the minimal JEPs helps reduce overlap in a set of patterns. Assume an operator $\operatorname{JEP}\left(D_{p}, D_{n}\right)$, which mines the minimal JEPs between $D_{p}$ and $D_{n}$. It can be computed by existing algorithms such as $[7,3,9]$, which can be treated as a "black box" ${ }^{2}$. The set of patterns which is output from mining may be used for either domain understanding or for the construction of models and (ensemble) classifiers. Although the set of mined patterns can be large, any specific test instance (transaction) is unlikely to match with many patterns from the ensemble. So with respect to any individual classification decision, the amount of redundancy amongst the matched patterns is likely to be small.

A data stream is a sequence of transactions. We define a sliding window of length $k$, which can be viewed as an evolving dataset. The window contains two sub-windows. The more recent sub-window is referred to as the positive class (i.e. $D_{p}$ ), and the less recent sub-window as the negative class (i.e. $D_{n}$ ), where $\left|D_{p}\right|+\left|D_{n}\right|=k$. See Fig. 1 for an illustration. In the current window

\footnotetext{
${ }^{2}$ In our experiments, we use an implementation of [3].
} 
(window $(i)$ ), as $\operatorname{JEP}\left(D_{p}, D_{n}\right)$ is being computed, newly arriving transactions are buffered (labeled as $\Delta_{p}$ ). When mining completes, the window is updated to include the buffered transactions. It now contains sub-windows new $D_{p}$ and $n e w D_{n}$, with the oldest transactions (labeled $\nabla_{n}$ ) being removed. At the same time, some transactions of $D_{p}$ are deleted from $D_{p}$ (labeled as $\nabla_{p}$ ) and inserted to new $D_{n}$ (labeled as $\Delta_{n}$ ). Thus, $\nabla_{p} \equiv \Delta_{n}$, new $D_{p}=D_{p}^{-} \cup \Delta_{p}$, and new $D_{n}=$ $D_{n}^{-} \cup \Delta_{n}$, where $D_{p}^{-}=D_{p}-\nabla_{p}$ and $D_{n}^{-}=D_{n}-\nabla_{n}$. The increment size, $\delta$, is the number of deleted and inserted transactions in each class, i.e. $\left|\nabla_{n}\right|=$ $\left|\Delta_{n}\right|=\left|\nabla_{p}\right|=\left|\Delta_{p}\right|=\delta$, and $\delta<<k$.

\section{Efficient Incremental JEP Maintenance Algorithms}

Our objective is to (incrementally) compute $\operatorname{JEP}\left(\right.$ new $D_{p}$, new $\left.D_{n}\right)$, given the inputs: $\operatorname{JEP}\left(D_{p}, D_{n}\right)$ and updates $\nabla_{p}, \nabla_{n}, \Delta_{p}, \Delta_{n}$. We will shortly describe our algorithm ExclusiveIncremental. First we provide some intuition about the limitations of the existing incremental technique from [10], known as LMDR.

LMDR processes the updates sequentially in four steps: It incrementally mines the patterns after each of the updates 1) $\nabla_{n}$, followed by 2) $\Delta_{n}$, then 3) $\nabla_{p}$ and then 4) $\Delta_{p}$. Patterns may be added at Steps 1) and 4), while existing patterns may be deleted at Steps 2) and 3). The drawback is that the effect of each update is evaluated independently of the other updates. This means that redundant computations may occur, whereby certain patterns appear due to an earlier update, only to be eliminated due to a later update (or vice versa).

For example, $\nabla_{n}$ and $\Delta_{n}$ may contain transactions which intersect. Consider two transactions $\{a, b, c, d\} \in \nabla_{n}$ and $\{c, d, e, f\} \in \Delta_{n}$. These intersect on the itemset $\{c, d\}$. Now $\{c, d\}$ might be a new pattern which is inserted after processing $\nabla_{n}$, but which then gets deleted after subsequently processing update $\Delta_{n}$. Similar interactions can occur with respect to the pairs of updates $\nabla_{n}$ plus 


\begin{tabular}{|c|c|}
\hline Algorithm 1 ExclusiveIncremental: & e contrasts w.r.t. $n e w D_{p}$ and $n e w D_{n}$ \\
\hline $\begin{array}{l}J_{0}=\operatorname{JEP}\left(D_{p}, D_{n}\right), D_{p}^{-}=D_{p}-\nabla_{p}, D_{n}^{-} \\
\text {new } D_{n}=D_{n}^{-} \cup \Delta_{n}\end{array}$ & $D_{n}-\nabla_{n}$, new $D_{p}=D_{p}^{-} \cup \Delta_{p}$ \\
\hline LMDR $^{+}$vs ExclusiveIncremental ${ }^{+}$ & new $\left.D_{p}, D_{n}\right)$ : compute $\operatorname{JEP}\left(\right.$ new $\left.D_{p}, D_{n}\right)$ \\
\hline$L M D R^{+}:$ & ExclusiveIncremental $^{+}:$ \\
\hline 1: $J_{\text {retain }} \leftarrow J_{0} \cap_{B} D_{p}^{-}$ & 1: $J_{\text {retain }}^{\prime} \leftarrow J_{0} \cap_{B}$ new $D_{p}$ \\
\hline 2: $J_{\text {appear }} \leftarrow \operatorname{JEP}\left(\Delta_{p}, D_{n}\right)$ & 2: $J_{\text {appear }}^{\prime} \leftarrow \operatorname{JEP}\left(\Delta_{p},\left(D_{p} \cup D_{n}\right)\right)$ \\
\hline 3: $\operatorname{JEP}\left(n e w D_{p}, D_{n}\right) \leftarrow J_{\text {retain }} \cup J_{\text {appear }}$ & 3: $\operatorname{JEP}\left(\right.$ new $\left.D_{p}, D_{n}\right) \leftarrow J_{\text {retain }}^{\prime} \cup J_{\text {appear }}^{\prime}$ \\
\hline LMDR $^{-}$vs ExclusiveIncremental ${ }^{-}$ &,$D_{p}$, new $\left.D_{n}\right):$ compute $\operatorname{JEP}\left(D_{p}\right.$, new $\left.D_{n}\right)$ \\
\hline$L M D R^{-}:$ & ExclusiveIncremental $^{-}:$ \\
\hline $\left.1: J_{n} \leftarrow J_{0} \cup\left(\operatorname{JEP}\left(\nabla_{n}, D_{n}^{-}\right) \cap_{B} D_{p}\right)\right)$ & 1: $J_{\text {invalid }} \leftarrow J_{0} \cap_{B} \Delta_{n}$ \\
\hline 2: $J_{\text {disappear }} \leftarrow \operatorname{JEP}\left(D_{p}, \Delta_{n}\right)$ & $2: J_{\text {retain }} \leftarrow J_{0}-J_{\text {invalid }}$ \\
\hline 3: $\operatorname{JEP}\left(D_{p}\right.$, new $\left.D_{n}\right) \leftarrow$ & 3: $J_{\text {new }} \leftarrow \operatorname{JEP}\left(\nabla_{n}\right.$, new $\left.D_{n}\right) \cap_{B} D_{p}$ \\
\hline$\left(J_{n} \times J_{\text {disappear }}\right) \cap_{B} D_{p}$ & 4: $J_{\text {disappear }} \leftarrow \operatorname{JEP}\left(D_{p}, \Delta_{n}\right)$ \\
\hline & $\begin{aligned} & \text { 5: } \operatorname{JEP}\left(D_{p}, \text { new } D_{n}\right) \leftarrow J_{\text {retain }} J_{\text {new }} \\
& \cup\left(\left(J_{\text {invalid }} \times J_{\text {disappear }}\right) \cap_{B} D_{p}\right)\end{aligned}$ \\
\hline ExclusiveIncremental $\left(J_{0}\right.$, new $D_{p}$, new & r): compute $\operatorname{JEP}\left(\right.$ new $D_{p}$, new $\left.D_{n}\right)$ \\
\hline 1: $J_{0}^{\prime} \leftarrow$ ExclusiveIncremental $^{-}\left(J_{0}, D_{p}^{-}\right.$ & $\left.e w D_{n}\right)$ \\
\hline 2: $\operatorname{JEP}\left(\right.$ new $D_{p}$, new $\left.D_{n}\right) \leftarrow$ ExclusiveIn & mental $^{+}\left(J_{0}^{\prime}\right.$, new $_{p}$, new $\left.D_{n}\right)$ \\
\hline
\end{tabular}

$\nabla_{p}$, and $\nabla_{p}$ plus $\Delta_{p}$. In contrast, our new algorithm avoids unnecessary work by taking into account the interactions between updates.

Our algorithm (ExclusiveIncremental) and a comparison to LMDR are shown in Algorithm 1. The logic is divided into two subalgorithms, one processing updates to $D_{n}$ and the other processing updates to $D_{p}$. ExclusiveIncremental is both correct (only mines valid patterns) and complete (mines all valid patterns).

Updating the Positive Class (ExclusiveIncremental ${ }^{+}$). Upon deletion of $\nabla_{p}, L M D R^{+}$keeps the old JEPs occurring in $D_{p}^{-}$(represented by $J_{\text {retain }}$, line 1). Subsequently, insertion of $\Delta_{p}$ may cause new JEPs to appear, which are JEPs for $\Delta_{p}$ versus $D_{n}$ (represented by $J_{\text {appear }}$, line 2 ). However, $J_{\text {retain }}$ and $J_{\text {appear }}$ may overlap, and so $J_{\text {appear }}$ may contain some old JEPs which were removed from $J_{\text {retain. }}$ To remove this cancellation, our algorithm finds both $J_{\text {retain }}^{\prime}$, which includes the old JEPs which occur in $\Delta_{p}$ (line 1$)$, and $J_{\text {appear }}^{\prime}$ which excludes new JEPs which occur in $D_{p}$ (line 2$) ;\left(J_{\text {retain }}^{\prime} \cap J_{\text {appear }}^{\prime}\right)=\emptyset$.

Updating the Negative Class (ExclusiveIncremental ${ }^{-}$). Upon deletion of $\nabla_{n}, L M D R^{-}$finds the newly occurring JEPs by finding itemsets of $\operatorname{JEP}\left(\nabla_{n}, D_{n}^{-}\right)$ 
which occur in $D_{p}$ (represented by $J_{n}$, line 1 ). Such itemsets do not exist in $J_{0}$. Then, the insertion of $\Delta_{n}$ is processed by finding the pair-wise union between $J_{n}$ and $J_{\text {disappear }}=\operatorname{JEP}\left(D_{p}, \Delta_{n}\right)$, to find all the minimal JEPs which occur in $D_{p}$, but not in $D_{n}^{-}$nor $\Delta_{n}$ (line 3 ). However, some itemsets in $J_{n}$ may remain in the output. They are itemsets which occur in $D_{p}$ but not in $\nabla_{n}$. Some of the old JEPs which were removed from $J_{n}$, moreover, may re-appear. This cancellation is removed in our algorithm. Firstly, all of the old JEPs which do not occur in $\Delta_{n}$ are kept (line 1-2). Then, the new JEPs are found from the deleted transactions in $\nabla_{n}$, which do not occur in $D_{n}^{-}$nor $\Delta_{n}$ (line 3). Finally, only old JEPs which occur in $\Delta_{n}$ (represented by $J_{\text {invalid }}$ ) are involved in the pair-wise union (line 5). $J_{\text {invalid }}$ is a smaller set than $J_{n}$.

Updating Both the Positive and Negative Classes (ExclusiveIncremental). When incremental changes occur to both classes, the two algorithms can be combined. We begin with ExclusiveIncremental ${ }^{-}()$for handling the changes in $D_{n}$, then pass its output to ExclusiveIncremental ${ }^{+}()$. Since $\nabla_{p}=$ $\Delta_{n}$ in a sliding window, references to $D_{p}$ in the first routine can be substituted by $D_{p}^{-}$. This avoids generating patterns which occur in $\nabla_{p}$. Line 1 in ExclusiveIncremental ${ }^{+}()$can be replaced by $J_{\text {retain }}^{\prime}=J_{0}$ for a sliding window.

\section{Performance Study and Discussion}

We compare the performance of our ExclusiveIncremental algorithm, against LMDR [10], and also a "Naive" algorithm which mines the JEPs from scratch in every window. We use three data stream datasets: DATA 1: KDD-CUP 1999, DATA 2: KDD-CUP 2000 and DATA 3: a synthetic dataset generated by the IBM data generator ${ }^{3}$. Tables 1 and 2 show the characteristics of each

\footnotetext{
${ }^{3}$ http://www.almaden.ibm.com. DATA 3 was generated with parameter '-g0 0.3 -p 10 shake 5'; -shake models the fuzzy boundary between the classes; $-g 0$ and $-p$ determine the class distribution. All continuous attributes are discretized by equi-width binning.
} 


\begin{tabular}{|r|rrr|}
\hline Dataset & DATA 1 & DATA 2 & DATA 3 \\
\hline Source & KDD'99 & KDD'00 & Synthetic \\
\#attributes & 20 & 30 & 10 \\
\#items & 200 & 600 & 50 \\
\hline
\end{tabular}

Table 1: Data Characteristics

\begin{tabular}{|r|rrrrr|}
\hline $\mathrm{S}$ & $\mathrm{A}$ & $\mathrm{B}$ & $\mathrm{C}$ & $\mathrm{D}$ & $\mathrm{E}$ \\
\hline$\left|D_{n}\right|$ & 1000 & 1000 & 5000 & 5000 & 10000 \\
$\left|D_{p}\right|$ & 200 & 200 & 500 & 1000 & 1000 \\
$\delta$ & 10 & 20 & 100 & 100 & 100 \\
\hline
\end{tabular}

Table 2: Scenarios $(\mathrm{S}=$ Scenario $)$

\begin{tabular}{|c|c|c|c|c|c|c|c|c|}
\hline Dataset & $\mathrm{S}$ & $\left|J_{0}\right|$ & \multicolumn{6}{|c|}{ Incremental Mining Time (seconds) } \\
\hline & & & \multicolumn{2}{|c|}{ PosIncremental } & \multicolumn{2}{|c|}{ NegIncremental } & \multicolumn{2}{|c|}{ PosNegIncremental } \\
\hline & & & Naive $^{+}$ & $\begin{array}{c}\text { ExInc }^{+} \\
\text {(speed-up) }\end{array}$ & Naive $^{-}$ & $\begin{array}{c}\text { ExInc } \\
\text { (speed-up) }\end{array}$ & Naive & $\begin{array}{c}\text { ExInc } \\
\text { (speed-up) }\end{array}$ \\
\hline \multirow[t]{5}{*}{ DATA 1} & $\overline{\mathrm{A}}$ & 10 & 0.05 & $0.01(5.00)$ & 0.12 & $0.04(3.00)$ & 0.17 & $0.01(17.00)$ \\
\hline & $\mathrm{B}$ & 2 & 0.02 & $0.02(1.00)$ & 0.12 & $0.17(0.71)$ & 0.14 & $\mathbf{0 . 0 3}(4.67)$ \\
\hline & $\mathrm{C}$ & 2 & 0.42 & $0.08(5.25)$ & 0.50 & $\mathbf{0 . 3 6}(1.39)$ & 0.92 & $0.15(6.13)$ \\
\hline & D & 5 & 0.28 & $\mathbf{0 . 0 7}(4.00)$ & 1.12 & $\mathbf{0 . 5 3}(2.11)$ & 1.40 & $\mathbf{0 . 2 1}(6.67)$ \\
\hline & $\mathrm{E}$ & 8 & 0.68 & $\mathbf{0 . 1 4}(4.86)$ & 2.45 & $1.02(2.40)$ & 3.13 & $\mathbf{0 . 2 6}(12.04)$ \\
\hline \multirow[t]{5}{*}{ DATA 2} & $\mathrm{~A}$ & 85 & 1.61 & $0.11(14.64)$ & 3.03 & $\mathbf{0 . 5 4}(5.61)$ & 4.64 & $\mathbf{0 . 1 9}(24.42)$ \\
\hline & B & 145 & 1.36 & $0.43(3.16)$ & 3.85 & $\mathbf{1 . 0 6}(3.63)$ & 5.21 & $\mathbf{0 . 5 9}(8.83)$ \\
\hline & $\mathrm{C}$ & 362 & 29.97 & $7.05(4.25)$ & 59.60 & $28.81(2.07)$ & 88.57 & $18.62(4.76)$ \\
\hline & D & 576 & 78.18 & $8.90(8.78)$ & 45.95 & $53.39(0.86)$ & 124.13 & $27.11(4.58)$ \\
\hline & $\mathrm{E}$ & 1005 & 63.18 & $\mathbf{1 5 . 4 7}(4.08)$ & 258.25 & $\mathbf{8 6 . 4 2}(2.99)$ & 321.43 & $\mathbf{3 7 . 6 4}(8.54)$ \\
\hline \multirow[t]{5}{*}{ DATA 3} & $\mathrm{~A}$ & 1333 & 0.30 & $1.47(0.20)$ & 1.15 & $0.49(2.35)$ & 1.45 & $\mathbf{1 . 9 1}(0.76)$ \\
\hline & B & 1357 & 0.32 & $2.69(0.12)$ & 1.17 & $1.00(1.17)$ & 1.49 & $3.06(0.49)$ \\
\hline & $\mathrm{C}$ & 1680 & 3.07 & $7.73(0.40)$ & 9.87 & $8.31(1.19)$ & 12.94 & $11.20(1.16)$ \\
\hline & $\mathrm{D}$ & 3149 & 11.41 & $12.38(0.92)$ & 20.24 & $16.66(1.21)$ & 31.65 & $22.22(1.42)$ \\
\hline & $\mathrm{E}$ & 2182 & 11.64 & $11.80(0.99)$ & 29.36 & $20.37(1.44)$ & 41.00 & $15.96(2.57)$ \\
\hline
\end{tabular}

Table 3: Runtime Comparison Against the Naive Algorithm; ExInc $=$ ExclusiveIncremental; speed-up $=\frac{\text { runtime of Naive }}{\text { runtime of ExclusiveIncremental }}$

data stream, and the scenarios used. All experiments used a $2.4 \mathrm{GHz} \mathrm{CPU}$ with 3GB RAM running Solaris. Tables 3 and 4 show the runtimes of each algorithm, averaged over 100 windows. ExInc refers to our ExclusiveIncremental algorithm. PosIncremental shows the runtimes for processing the positive class changes, NegIncremental for processing the negative class changes, and PosNegIncremental for processing simultaneous changes to both classes.

Compared to Naive, our algorithm is usually faster, depending (as expected) on the degree of change undergone by the window. For handling the changes in the positive class, ExInc ${ }^{+}$is up to 8 times faster than Naive $^{+}$, for which $D_{p}$ and $D_{n}$ have a large size and the increment is small, and it is up to 3 times faster than $L M D R^{+}$for DATA 1 and DATA 2. For DATA 3 however, since it is dense (i.e. contains a small number of items but a large number of patterns), ExInc ${ }^{+}$is slower than both Naive $^{+}$and $L M D R^{+}$, except for scenario D where the number 


\begin{tabular}{|c|c|c|c|c|c|c|c|}
\hline \multirow[t]{3}{*}{ Dataset } & $\mathrm{S}$ & \multicolumn{6}{|c|}{ Incremental Mining Time (seconds) } \\
\hline & & \multicolumn{2}{|c|}{ PosIncremental } & \multicolumn{2}{|c|}{ NegIncremental } & \multicolumn{2}{|c|}{ PosNegIncremental } \\
\hline & & $L M D R^{+}$ & $\begin{array}{c}\text { ExInc }^{+} \\
\text {(speed-up) }\end{array}$ & $L M D R^{-}$ & $\begin{array}{c}\text { ExInc }^{-} \\
\text {(speed-up) }\end{array}$ & $L M D R$ & $\begin{array}{c}\text { ExInc } \\
\text { (speed-up) }\end{array}$ \\
\hline \multirow[t]{5}{*}{ DATA 1} & A & 0.01 & $\mathbf{0 . 0 1}(1.00)$ & 0.06 & $\mathbf{0 . 0 4}(1.50)$ & 0.07 & $\mathbf{0 . 0 1}(7.00)$ \\
\hline & B & 0.02 & $0.02(1.00)$ & 0.01 & $0.17(0.06)$ & 0.03 & $0.03(1.00)$ \\
\hline & $\mathrm{C}$ & 0.19 & $\mathbf{0 . 0 8}(2.38)$ & 0.23 & $0.36(0.64)$ & 0.42 & $\mathbf{0 . 1 5}(2.80)$ \\
\hline & $\mathrm{D}$ & 0.19 & $\mathbf{0 . 0 7}(2.71)$ & 0.20 & $0.53(0.38)$ & 0.39 & $0.21(1.86)$ \\
\hline & $\mathrm{E}$ & 0.44 & $0.14(3.14)$ & 0.33 & $1.02(0.32)$ & 0.77 & $\mathbf{0 . 2 6}(2.96)$ \\
\hline \multirow[t]{5}{*}{ DATA 2} & $\overline{\mathrm{A}}$ & 0.27 & $\mathbf{0 . 1 1}(2.45)$ & 20.61 & $0.54(38.17)$ & 20.88 & $\mathbf{0 . 1 9}(109.89)$ \\
\hline & B & 0.82 & $0.43(1.91)$ & 78.42 & $1.06(73.98)$ & 79.24 & $0.59(134.31)$ \\
\hline & $\mathrm{C}$ & 24.34 & $7.05(3.45)$ & 871.13 & $28.81(30.24)$ & 895.47 & $\mathbf{1 8 . 6 2}(43.26)$ \\
\hline & $\mathrm{D}$ & 28.55 & $8.90(3.21)$ & 1482.7 & $\mathbf{5 3 . 3 9}(27.77)$ & 1511.3 & $27.11(55.75)$ \\
\hline & $\mathrm{E}$ & 52.01 & $\mathbf{1 5 . 4 7}(3.36)$ & 3217.8 & $\mathbf{8 6 . 4 2}(37.23)$ & 3269.8 & $\mathbf{3 7 . 6 4}(86.87)$ \\
\hline \multirow[t]{5}{*}{ DATA 3} & $\mathrm{~A}$ & 1.42 & $1.47(0.97)$ & 1255.3 & $\mathbf{0 . 4 9}(2561.86)$ & 1256.7 & $\mathbf{1 . 9 1 ( 6 5 7 . 9 7 )}$ \\
\hline & B & 2.18 & $2.69(0.81)$ & 5512.7 & $\mathbf{1 . 0 0}(5512.70)$ & 5514.9 & $3.06(1802.25)$ \\
\hline & $\mathrm{C}$ & 5.78 & $7.73(0.75)$ & 22441 & $\mathbf{8 . 3 1}(2700.48)$ & 22447 & $\mathbf{1 1 . 2 0}(2004.11)$ \\
\hline & $\mathrm{D}$ & 17.89 & $\mathbf{1 2 . 3 8}(1.45)$ & 40225 & $\mathbf{1 6 . 6 6}(2414.47)$ & 40243 & $\mathbf{2 2 . 2 2}(1811.12)$ \\
\hline & $\mathrm{E}$ & 11.18 & $11.80(0.95)$ & 49010 & $\mathbf{2 0 . 3 7}(2405.99)$ & 49021 & $\mathbf{1 5 . 9 6}(3071.49)$ \\
\hline
\end{tabular}

Table 4: Runtime Comparison Against the LMDR [10] algorithm; ExInc = ExclusiveIncremental; speed-up $=\frac{\text { runtime of } L M D R}{\text { runtime of ExclusiveIncremental }}$

of patterns is the largest. The results also show that $L M D R^{+}$performs better than Naive ${ }^{+}$in all scenarios for DATA 1 and DATA 2.

For processing changes in the negative class, ExInc ${ }^{-}$is up to 5 times faster than Naive $^{-}$, but $L M D R^{-}$is the fastest in most scenarios for DATA 1, which is sparse and contains less patterns. ExInc ${ }^{-}$is less efficient in this scenario. When the number of patterns is large, such as in DATA 2 and DATA $3, L M D R^{-}$is slower than Naive $^{-}$, and ExInc ${ }^{-}$is up to 5000 times faster than $L M D R^{-}$, especially for the dense DATA 3. In those scenarios, the pair-wise union operation performed by $L M D R^{-}$(line 3 in Algorithm 1 ) is computationally expensive, due to the large number of patterns involved.

Finally, for handling changes in both classes, ExInc has the fastest runtimes, followed by LMDR, and Naive, for DATA 1. For DATA 2 and DATA 3, where $L M D R$ performs poorly because of the slow runtime of $L M D R^{-}$, ExInc is able to outperform LMDR by up to 5000 times. 


\section{References}

[1] Aggarwal, C. C., 2003. A framework for diagnosing changes in evolving data streams. In: Int'l Conf. on Management of Data. San Diego, CA, pp. 575-586.

[2] Alhammady, H., Ramamohanarao, K., 2005. Mining emerging patterns and classification in data streams. In: Int'l Conference on Web Intelligence. pp. 272-275.

[3] Bailey, J., Manoukian, T., Ramamohanarao, K., 2003. A fast algorithm for computing hypergraph transversals and its application in mining emerging patterns. In: Proc. 3rd ICDM. Melbourne, Florida, pp. 485-488.

[4] Ben-David, S., Gehrke, J., Kifer, D., 2004. Detecting change in data streams. In: Proc. 30th VLDB Conference. Toronto, Canada, pp. 180-191.

[5] Chang, J. H., Lee, W. S., 2004. A sliding window method for finding recently frequent itemsets over online data streams. J. of Information Science and Engineering 20, 753-762.

[6] Dong, G., et al., 2003. Online mining of changes from data streams. In: Workshop on Management and Processing of Data Streams.

[7] Dong, G., Li, J., 1999. Efficient mining of emerging patterns: Discovering trends and differences. In: Proc. of KDD. San Diego, CA, pp. $43-52$.

[8] Dong, G., Zhang, X., Wong, L., Li, J., 1999. Caep: Classification by aggregating emerging patterns. In: Discovery Science. pp. 30-42.

[9] Li, J., Liu, G., Wong, L., 2007. Mining statistically important equivalence classes and delta-discriminative emerging patterns. In: Proc. of KDD. pp. 430-439.

[10] Li, J., Manoukian, T., Dong, G., Ramamohanarao, K., 2004. Incremental maintenance on the border of the space of emerging patterns. DMKD 9 (1), 89-116.

[11] Li, J., Ramamohanarao, K., Dong, G., 2000. Making use of the most expressive jumping emerging patterns for classification. In: Proc. 4th PAKDD. Kyoto, Japan, pp. 220-232. 